\title{
Direct comparison of predictive performance of PRECISE- DAPT versus PARIS versus CREDO-Kyoto: a subanalysis of the ReCre8 trial
}

\author{
R. Rozemeijer (D) - W. P. van Bezouwen - N. D. van Hemert · J. A. Damen · S. Koudstaal · M. Stein · G. E. Leenders · \\ L. Timmers · A. O. Kraaijeveld · K. Roes · P. Agostoni · P. A. Doevendans · P. R. Stella · M. Voskuil
}

Published online: 21 September 2020

(C) The Author(s) 2020

\begin{abstract}
Background Multiple scores have been proposed to guide risk stratification after percutaneous coronary intervention. This study assessed the performance of the PRECISE-DAPT, PARIS and CREDO-Kyoto risk scores to predict post-discharge ischaemic or bleeding events.

Methods A total of 1491 patients treated with latest-generation drug-eluting stent implantation were evaluated. Risk scores for post-discharge ischaemic or bleeding events were calculated and directly compared. Prognostic performance of both risk scores was assessed with calibration, Harrell's c-statistics net reclassification index and decision curve analyses.

Results Post-discharge ischaemic events occurred in 56 patients $(3.8 \%)$ and post-discharge bleeding events in 34 patients $(2.3 \%)$ within the first year after the invasive procedure. C-statistics for the PARIS ischaemic risk score was marginal $(0.59,95 \%$ confidence interval
\end{abstract}

W.P. van Bezouwen and N.D. van Hemert contributed equally and share second authorship.

Electronic supplementary material The online version of this article (https://doi.org/10.1007/s12471-020-01486-y) contains supplementary material, which is available to authorized users.

R. Rozemeijer $(\bowtie) \cdot$ W. P. van Bezouwen · N. D. van Hemert .

S. Koudstaal · M. Stein · G. E. Leenders · L. Timmers ·

A. O. Kraaijeveld · P. A. Doevendans · P. R. Stella · M. Voskuil

Department of Cardiology, University Medical Center Utrecht, Utrecht, The Netherlands

r.rozemeijer@umcutrecht.nl

\section{J. A. Damen}

Julius Center for Health Sciences and Primary Care, University Medical Center Utrecht, Utrecht, The Netherlands

\section{S. Koudstaal}

Farr Institute of Health Informatics, University College

London, London, UK
(CI) 0.51-0.68), whereas the CREDO-Kyoto ischaemic risk score was moderate $(0.68,95 \%$ CI $0.60-0.75)$. With regard to post-discharge bleeding events, CREDOKyoto displayed moderate discrimination (c-statistic 0.67, 95\% CI 0.56-0.77), whereas PRECISE-DAPT (0.59, 95\% CI 0.48-0.69) and PARIS (0.55, 95\% CI 0.44-0.65) had a marginal discriminative capacity. Net reclassification index and decision curve analysis favoured CREDO-Kyoto-derived bleeding risk assessment.

Conclusion In this contemporary all-comer population, PARIS and PRECISE-DAPT risk scores were not resilient to independent testing for post-discharge bleeding events. CREDO-Kyoto-derived risk stratification was associated with a moderate predictive capability for post-discharge ischaemic or bleeding events. Future studies are warranted to improve risk stratification with more focus on robustness and rigorous testing.

\footnotetext{
M. Stein

Department of Cardiology, Zuyderland Medical Center, Heerlen, The Netherlands

L. Timmers

St. Antonius Hospital, Nieuwegein, The Netherlands

K. Roes

Department of Biostatistics and Research Support, University Medical Center Utrecht, Utrecht, The Netherlands

P. Agostoni

Department of Cardiology, Hartcentrum, Ziekenhuis Netwerk Antwerpen Middelheim, Antwerp, Belgium

P. A. Doevendans

Netherlands Heart Institute, Utrecht, The Netherlands

Central Military Hospital, Utrecht, The Netherlands
} 
Keywords Coronary artery disease $\cdot$ Risk stratification · Bleeding · Thrombosis · Percutaneous coronary intervention

\section{Introduction}

Dual antiplatelet therapy (DAPT), consisting of aspirin and a $\mathrm{P}_{2} \mathrm{Y}_{12}$ inhibitor, represents the cornerstone of treatment for patients with acute coronary syndrome or after percutaneous coronary intervention (PCI) with drug-eluting stent implantation [1-3]. DAPT mitigates the risk of ischaemic events [4], but this is counterbalanced by an increased risk of bleeding events [5], mainly gastro-intestinal bleeding.

The risk scores of PRECISE-DAPT [6], PARIS [7] and CREDO-Kyoto [8] have been developed specifically to assess the risks of both post-discharge ischaemic and bleeding events following PCI. Although all three risk scores were moderately accurate in their derivation cohorts (c-statistics of $\sim 0.65$ to 0.70 ), they remain poorly characterised in external cohorts. Indeed, the prognostic performance has not yet been directly compared. Accordingly, we aimed to assess and to directly compare the predictive performance of currently used risk scores for post-discharge ischaemic or bleeding events in a contemporary all-comer population.

\section{Methods}

\section{Study design and patient population}

The present study is a subanalysis of the physicianinitiated, prospective, multicentre Randomised AllComers Evaluation of a Permanent Polymer Zotarolimus-Eluting Stent Versus a Polymer-Free Amphilimus-Eluting Stent (ReCre8) trial, as previously reported $[9,10]$. In brief, the ReCre8 trial was designed to evaluate clinical non-inferiority of the polymer-free amphilimus-eluting stent as compared with a latest-generation permanent polymer zotarolimuseluting stent in a 1:1 ratio across three European centres. Between 3 November, 2014 and 10 July, 2017, consecutive patients were randomly allocated to a stent group after stratification for troponin status and presence of diabetes mellitus. In both treatment arms, troponin-positive patients were planned for 12-month DAPT, whereas troponin-negative patients were planned for 1-month DAPT. Inclusion criteria were broad, while exclusion criteria were minor to reflect routine clinical practice.

The protocol was approved by the Medical Research Ethics Committee Utrecht and the institutional review board of each participating centre and monitored by an independent clinical research organisation (Julius Clinical Research, Zeist, the Netherlands). Clinical endpoints were defined according to the Academic Research Consortium criteria [11] and Bleeding Academic Research Consortium criteria [12] and adju- dicated by an independent clinical event committee, with complete verification of source documents. Postdischarge events were defined as adverse events occurring 2 or more days after the index procedure. This study complied with the principles of the Declaration of Helsinki and was reported according to the Transparent Reporting of a multivariable prediction model for Individual Prognosis Or Diagnosis (TRIPOD) statement [13]. Written informed consent was obtained from each patient that participated in this study.

\section{Risk scores}

The PRECISE-DAPT [6], PARIS [7] and CREDO-Kyoto risk scores [8] were calculated and assigned to each patient using established definitions (see files in the Electronic Supplementary Material: Tab. 1 and 2 and Fig. 1). To enable comparisons between the PRECISEDAPT and the other risk scores, we categorised patients into three risk strata (i.e. low, intermediate and high risk) by considering 'very low risk' and 'low risk' as one risk stratum. Creatinine clearance was calculated using the Cockcroft-Gault formula [14]. Anaemia was defined as a haemoglobin level $<7.0 \mathrm{mmol} / \mathrm{L}$ for women and $<7.5 \mathrm{mmol} / \mathrm{L}$ for men [15].

\section{Statistical analysis}

Continuous variables are expressed as mean \pm standard deviation (SD), and binary variables as counts $(n)$ and percentages (\%). Differences were tested using the Wilcoxon rank-sum test and the Student's $t$-test for continuous variables, or the $\chi^{2}$ test as appropriate. First, risk score distributions were visualised graphically. Patients were categorised into different risk strata (i.e. low, intermediate and high risk). The

\section{What's new?}

- Currently available risk scores may improve stratification for the risk of post-discharge events. Performance data and analyses with a direct comparison of risk scores are, however, limited.

- We used a contemporary cohort of patients to calculate three risk scores to compare net reclassification indices, receiver operating characteristic curves and decision curves.

- CREDO-Kyoto-derived risk stratification had a moderate predictive performance for post-discharge ischaemic or bleeding events.

- PARIS-derived and PRECISE-DAPT-derived risk stratifications were not resilient to independent testing for the risk of post-discharge events.

- Future studies are warranted to improve risk stratification with focus on risk score robustness and rigorous testing in external datasets. 
Table 1 Baseline characteristics of ReCre8 trial population, compared with derivation cohorts for PARIS, CREDO-Kyoto and PRECISE-DAPT risk scores

\begin{tabular}{|c|c|c|c|c|c|c|c|}
\hline Characteristic & $\begin{array}{l}\text { ReCre8 } \\
(n=1491)\end{array}$ & $\begin{array}{l}\text { PARIS } \\
(n=4190)\end{array}$ & $p$-value ${ }^{\mathrm{a}}$ & $\begin{array}{l}\text { CRED0-Kyoto } \\
(n=4778)\end{array}$ & $p$-value ${ }^{b}$ & $\begin{array}{l}\text { PRECISE-DAPT } \\
(n=14,963)\end{array}$ & $p$-value ${ }^{c}$ \\
\hline \multicolumn{8}{|l|}{ Clinical characteristics } \\
\hline Age, years & $64.9 \pm 11.0$ & $63.6 \pm 11.0$ & 0.50 & $68.1 \pm 10.3$ & $<0.001$ & $65.0(56.9-73.0)$ & - \\
\hline Female & $349(23.4)$ & $1072(25.4)$ & 0.088 & $1331(27.8)$ & $<0.001$ & $4414(29.5)$ & $<0.001$ \\
\hline Body mass index, $\mathrm{kg} / \mathrm{m}^{2}$ & $27.3 \pm 4.4$ & $29.3 \pm 5.5$ & $<0.001$ & $23.8 \pm 3.4$ & $<0.001$ & $\mathrm{~N} / \mathrm{A}$ & - \\
\hline Current smoker & $384(25.8)$ & 745 (17.8) & $<0.001$ & $1322(27.6)$ & 0.15 & $3757(28.0)$ & - \\
\hline Diabetes mellitus & $304(20.4)$ & $1422(34.1)$ & $<0.001$ & $1952(40.9)$ & $<0.001$ & $4168(27.9)$ & $<0.001$ \\
\hline Insulin-treated & $96(6.4)$ & $473(11.2)$ & $<0.001$ & $499(10.4)$ & $<0.001$ & $797(5.4)$ & 0.24 \\
\hline $\begin{array}{l}\text { Low eGFR }(<60 \mathrm{ml} / \mathrm{min} \text { per } \\
\left.1.73 \mathrm{~m}^{2}\right)^{\mathrm{d}}\end{array}$ & $164(11.0)$ & $663(15.8)$ & $<0.001$ & $374(7.9)^{\mathrm{e}}$ & $<0.001$ & $\mathrm{~N} / \mathrm{A}$ & - \\
\hline WBC count ( $10^{3}$ units $\left./ \mu \mathrm{L}\right)$ & $8.2(6.6-10.6)$ & $\mathrm{N} / \mathrm{A}$ & - & $N / A$ & - & $7.8(6.3-10.2)$ & - \\
\hline Anaemia $^{f}$ & $97(6.5)$ & $653(15.5)$ & $<0.001$ & $517(10.8)$ & $<0.001$ & $\mathrm{~N} / \mathrm{A}$ & - \\
\hline $\begin{array}{l}\text { Low platelet count } \\
\left(<100 \times 10^{9} / \mathrm{L}\right)\end{array}$ & $11(0.7)$ & $\mathrm{N} / \mathrm{A}$ & - & $63(1.3)$ & 0.089 & $\mathrm{~N} / \mathrm{A}$ & - \\
\hline Triple therapy & $120(8.0)$ & $202(4.8)$ & $<0.001$ & $389(8.1)$ & 0.90 & $\mathrm{~N} / \mathrm{A}$ & - \\
\hline Impaired LVEF & $278(18.6)$ & $\mathrm{N} / \mathrm{A}$ & - & $772(16.2)$ & 0.06 & $N / A$ & - \\
\hline Peripheral vascular disease & $\mathrm{N} / \mathrm{A}$ & $334(8.0)$ & - & $371(7.8)$ & - & $714(10.4)$ & - \\
\hline Malignancy & $\mathrm{N} / \mathrm{A}$ & $\mathrm{N} / \mathrm{A}$ & - & $408(8.5)$ & - & $\mathrm{N} / \mathrm{A}$ & - \\
\hline \multicolumn{8}{|l|}{ Relevant medical history } \\
\hline Prior Ml & $297(19.9)$ & $1044(24.9)$ & $<0.001$ & $641(13.4)$ & $<0.001$ & $2946(19.8)$ & 0.86 \\
\hline Prior PCl & $304(20.4)$ & $1758(41.9)$ & $<0.001$ & $0(0)$ & - & $2392(16.0)$ & 0.54 \\
\hline Prior CABG & $138(9.3)$ & $603(14.3)$ & $<0.001$ & $0(0)$ & - & $893(6.0)$ & $<0.001$ \\
\hline Prior bleeding ${ }^{g}$ & $19(1.3)$ & $\mathrm{N} / \mathrm{A}$ & - & N/A & - & $82(1.9)$ & 0.001 \\
\hline \multicolumn{8}{|l|}{ Clinical presentation } \\
\hline Stable angina & $633(42.2)$ & $2622(62.5)$ & $<0.001$ & $N / A$ & - & $6299(44.4)$ & 0.98 \\
\hline Unstable angina & $109(7.2)$ & 1254 (29.9) & $<0.001$ & N/A & - & $3215(22.7)$ & $<0.001$ \\
\hline NSTEMI or STEMI & 599 (40.2) & $331(7.8)$ & $<0.001$ & $733(15.3)$ & $<0.001$ & 4669 (32.2) & $<0.001$ \\
\hline \multicolumn{8}{|l|}{ Procedural features } \\
\hline Chronic total occlusion & $98(6.6)$ & $\mathrm{N} / \mathrm{A}$ & - & 633 (13.2) & $<0.001$ & $\mathrm{~N} / \mathrm{A}$ & - \\
\hline $\begin{array}{l}\text { Data are } n(\%) \text {, mean } \pm \text { stanc } \\
C A B G \text { coronary artery bypas } \\
\text { available, NSTEMI non-ST-se } \\
W B C \text { white blood cell } \\
{ }^{a} P \text {-value for comparison of } \mathrm{R} \\
{ }^{b} P \text {-value for comparison of } \mathrm{R} \\
{ }^{c} P \text {-value for comparison of } \mathrm{R} \\
{ }^{\mathrm{d}} \text { eGFR was calculated using } \\
\text { e Defined as eGFR }<30 \mathrm{ml} / \mathrm{mi} \\
{ }^{\mathrm{f}} \text { Defined as haemoglobin lev } \\
{ }^{9} \text { Defined as history of previo }\end{array}$ & $\begin{array}{l}\text { deviation, or media } \\
\text { afting, eGFR estim } \\
\text { nt elevation myoca } \\
8 \text { and PARIS cohor } \\
8 \text { and CREDO-Kyot } \\
8 \text { and PRECISE-DA } \\
\text { ockcroft-Gault forn } \\
1.73 \mathrm{~m}^{2} \\
7.0 \mathrm{mmol} / \mathrm{L} \text { for wor }\end{array}$ & $\begin{array}{l}\text { (interquartile ra } \\
\text { ted glomerular } \mathrm{f} \\
\text { dial infarction, } P \\
\text { s cohorts } \\
\text { T cohorts } \\
\text { iula } \\
\text { en and }<7.5 \mathrm{mn}\end{array}$ & $\begin{array}{l}\text { e) } \\
\text { percutaneo }\end{array}$ & $\begin{array}{l}\text { VEF left ventricul } \\
\text { coronary interve }\end{array}$ & $\begin{array}{l}\text { tion fractio } \\
\text { STEMIST-s }\end{array}$ & $\begin{array}{l}\text { MI myocardial infarc } \\
\text { ment elevation myoc }\end{array}$ & $\begin{array}{l}\text { N/A not } \\
\text { I infarction, }\end{array}$ \\
\hline
\end{tabular}

ability to discriminate between low- and high-risk strata for both risk scores regarding post-discharge ischaemic or bleeding events was evaluated using Kaplan-Meier estimates of time to first post-discharge event with log-rank tests [16].

Indices of calibration and discrimination were used to assess predictive performance. Calibration (i.e. the degree to which the estimated risks match the observed risks) was assessed and plotted as observed versus predicted outcomes for each risk stratum of each risk score. To assess model discrimination (i.e. the ability to distinguish patients at low, intermediate or high risk), receiver operating characteristic curves and Harrell's c-statistics with 95\% confidence inter- val (CI) were calculated and compared using the nonparametric approach of DeLong [17]. The net reclassification improvement index was calculated and decision curve analysis [18] was used to determine a possible net benefit (i.e. the balance between the number of true positives and false positives) over a range of cut-off values: the higher the net benefit, the better the risk score. Theoretically, cut-off values should range from negative infinity to the incidence of the disease or outcome of interest.

A complete-case analysis was performed since the number of missing values was low $(<2.5 \%)$. $P$-values were two-sided and were considered statistically significant when $p<0.05$. Statistical analyses were 
Fig. 1 Distribution of contemporary risk scores. Risk strata were evaluated using $\chi^{2}$ test, with established definitions [6-8]
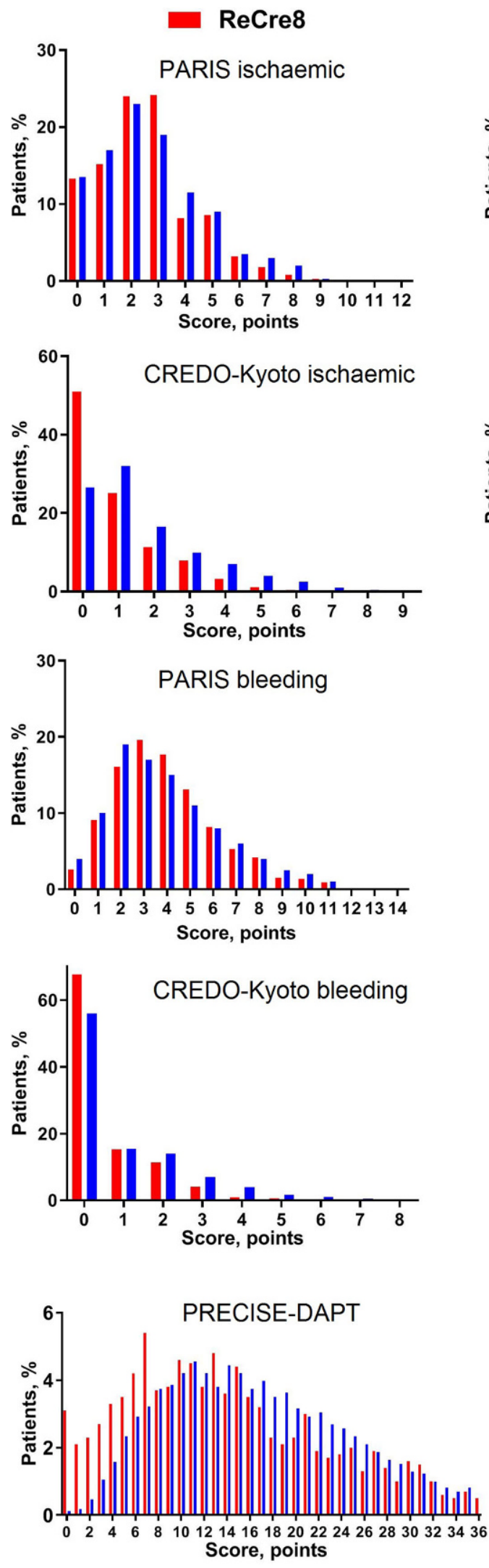
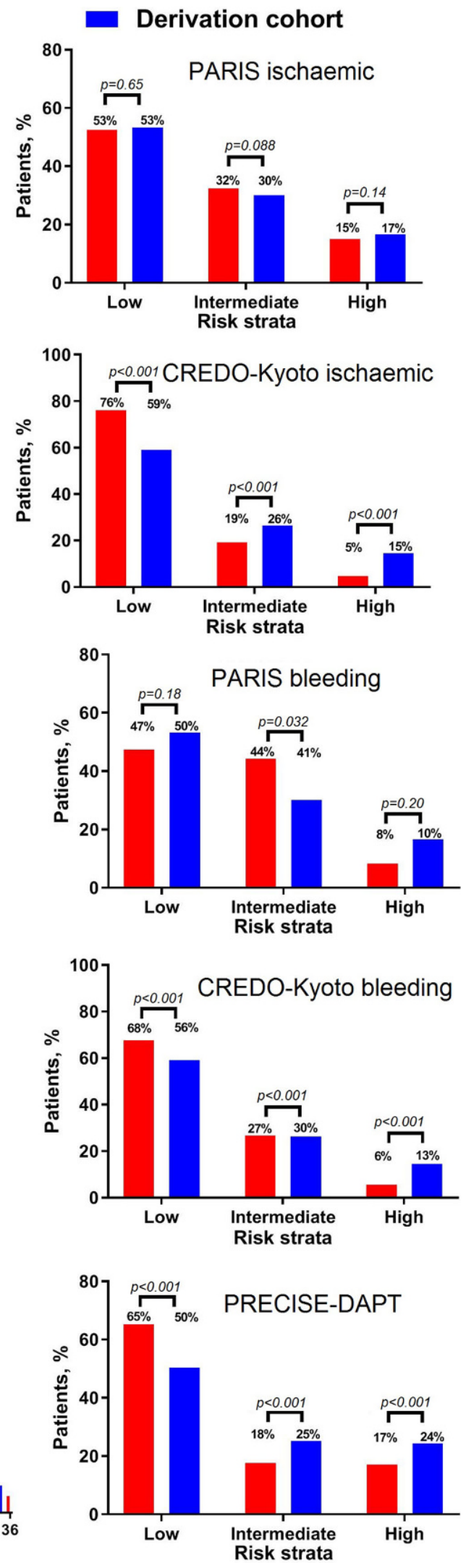
Hier steht eine Anzeige.

Springer 
Hier steht eine Anzeige.

Springer 
Hier steht eine Anzeige.

Springer 
Hier steht eine Anzeige.

Springer 
Fig. 2 One-year KaplanMeier curves for various risk strata using contemporary risk scores. IM intermediate, $P D-B E$ postdischarge bleeding events, $P D$-IE post-discharge ischaemic events
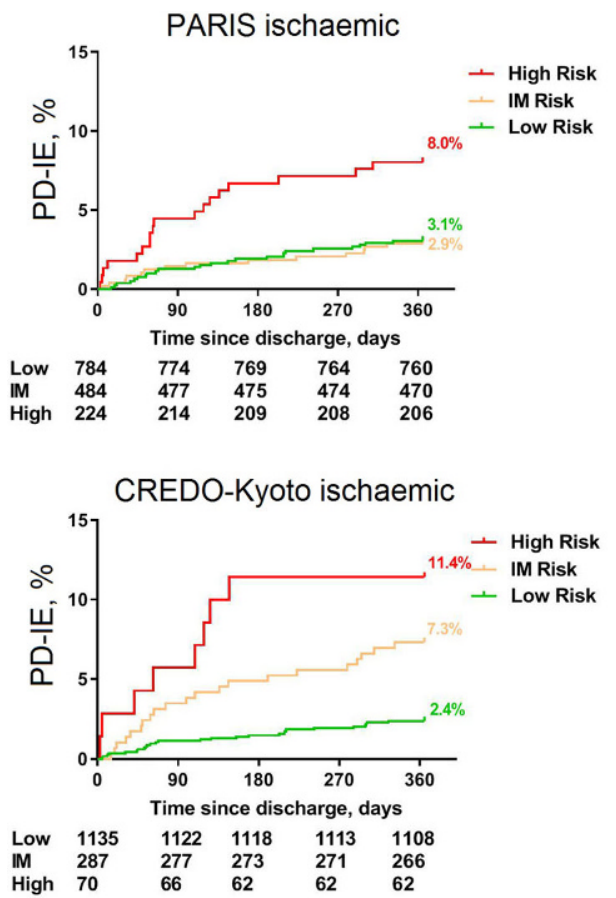
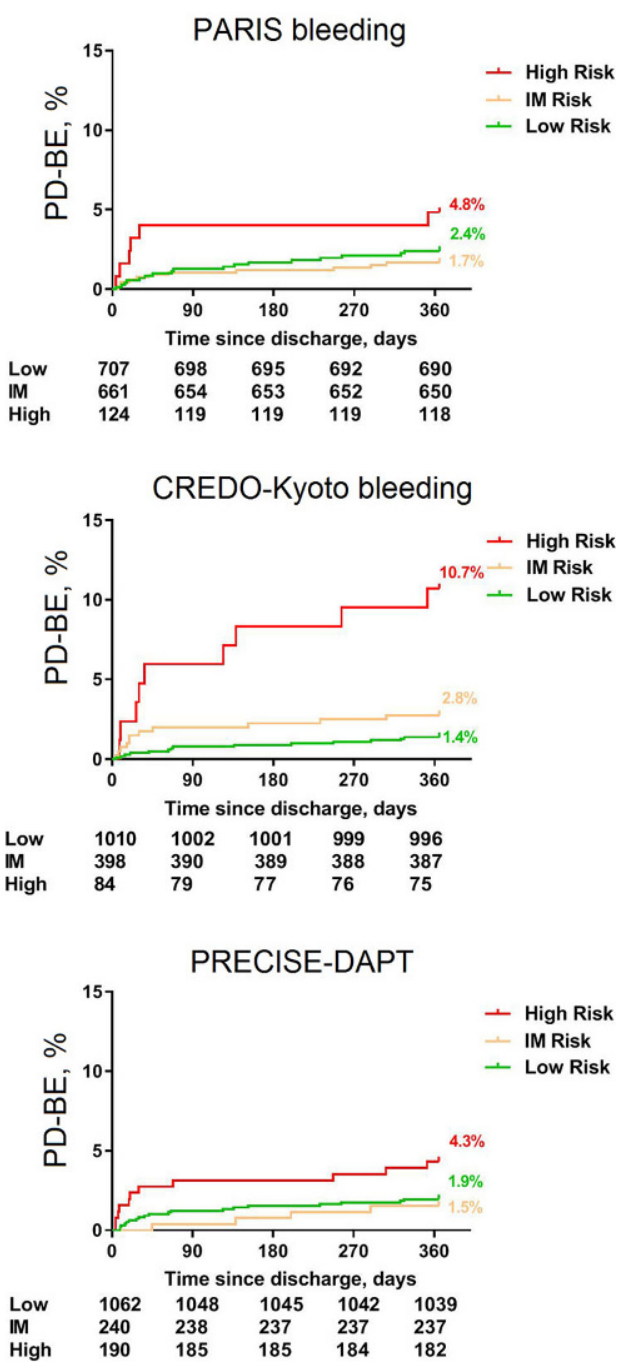

performed using SAS version 9.4 (SAS Institute, Cary, USA) and R version 3.4.1 (R Foundation for Statistical Computing, Vienna, Austria). Figures were generated using GraphPad Prism version 7.04 (GraphPad Inc., San Diego, CA, USA) and R version 3.4.1.

\section{Results}

\section{Baseline characteristics and risk score distribution}

The ReCre8 trial population comprised 1491 patients and was characterised by a lower number of diabetic patients (20\% vs $34 \%, 41 \%$ and $28 \%)$ and a higher number of troponin-positive acute coronary syndrome patients $(40 \%$ vs $8 \%, 15 \%$ and $32 \%)$ than the derivation cohorts of the PARIS, CREDO-Kyoto and PRECISE-DAPT risk scores (Tab. 1). Compared with the PARIS cohort, current smoking was more prevalent ( $25.8 \%$ vs $17.8 \%)$, but anaemia was less prevalent (6.5\% vs $15.5 \%)$. Fewer patients had renal insufficiency ( $11.0 \%$ vs $15.8 \%)$. Compared with the CREDOKyoto cohort, more patients in the ReCre8 trial had a history of myocardial infarction (19.9\% vs $13.4 \%)$.
The baseline characteristics of the PRECISE-DAPT cohort were generally similar, although the ReCre8 trial population had a lower incidence of prior bleeding (1.3\% vs $1.9 \%)$.

The risk score distributions in our cohort were generally comparable to those of the derivation cohorts of the PARIS, CREDO-Kyoto and PRECISE-DAPT risk scores (Fig. 1). The proportion of patients that were classified into the low, intermediate and high ischaemic risk strata using the PARIS ischaemic risk score in the ReCre8 trial population were similar to the PARIS derivation cohort. Conversely, using PRECISEDAPT to stratify our trial population, less patients were categorised as low risk $(65 \%$ vs $50 \%)$ or high risk ( $17 \%$ vs $24 \%$ ) compared with the PRECISE-DAPT derivation cohort.

\section{Post-discharge adverse events}

One-year Kaplan-Meier curves assessing the divergence of post-discharge ischaemic events over the risk strata showed significant differences for PARIS (logrank $p<0.001$ ), CREDO-Kyoto (log-rank $p<0.001)$ and 


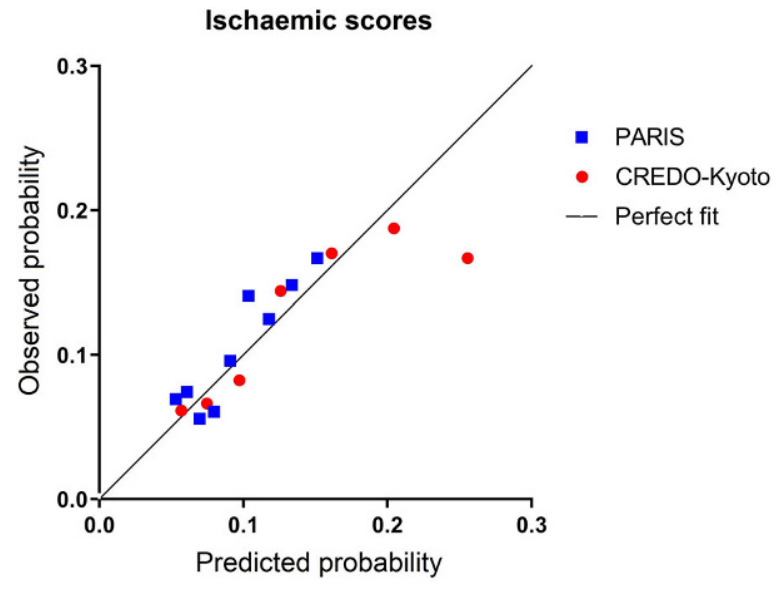

Fig. 3 Expected and observed rates of post-discharge events using contemporary risk scores. A perfect fit (diagonal line) represents predicted probability being equal to observed probability. Points under this line (predicted proba-

PRECISE-DAPT (log-rank $p<0.001$ ) (Fig. 2). However, some overlap was seen in the low- and intermediaterisk stratum using the PARIS ischaemic risk score and the PRECISE-DAPT risk score. In the present study post-discharge ischaemic events occurred in 56 patients $(3.8 \%)$ and post-discharge bleeding events in 34 patients $(2.3 \%)$.

Cardiac death occurred in 18 patients (1.2\%), 14 patients suffered a myocardial infarction (0.9\%), 6 had a definite or probable stent thrombosis $(0.4 \%)$ and 8 had an ischaemic stroke $(0.5 \%)$. Post-discharge bleeding events occurred in 34 patients $(2.3 \%)$ within the first year after the index PCI.

\section{Predictive performance of ischaemic risk scores}

Calibration of the ischaemic risk scores appeared reasonable for both PARIS and CREDO-Kyoto with regard to the predicted post-discharge ischaemic event rates (Fig. 3). Discriminative power and reclassification are summarised in Tab. 2. Discriminative performance of PARIS (Fig. 4a) was marginal (c-statistic 0.59, 95\% CI 0.51-0.68), whereas that of CREDO-Kyoto was moderate $(0.68,95 \%$ CI $0.60-0.75)$. Reclassification with CREDO-Kyoto resulted in a lower number of cases in the high-risk stratum (i.e. fewer patients with postdischarge ischaemic events were classified in highrisk categories by CREDO-Kyoto) than with PARIS (see Tab. 4 in the Electronic Supplementary Material).

CREDO-Kyoto was able to enhance risk stratification on post-discharge ischaemic events in all-comer patients treated with latest-generation drug-eluting stent implantation as compared with the situation in which no model was used (Fig. 4b). When compared with the situation of assuming all patients as low risk, CREDO-Kyoto enhanced stratification at a risk threshold of $\geq 2.0 \%$. Applying CREDO-Kyoto when using a risk threshold of $4.0 \%$ resulted in a net benefit of $+1.0 \%$, while assuming all patients as high risk

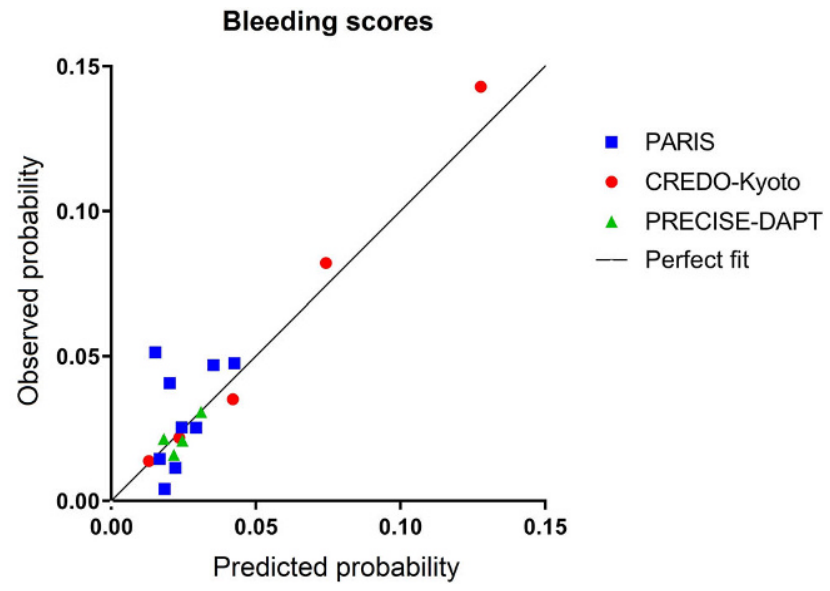

bility > observed probability) indicate an overestimation of the risk of post-discharge events, while points above this line (predicted probability > observed probability) indicate an underestimation of the risk of post-discharge events

resulted in a net benefit of $-0.3 \%$; for PARIS, this resulted in a net benefit of $+0.5 \%$ (see Tab. 5 in the Electronic Supplementary Material). In other words, the net benefit using CREDO-Kyoto-derived risk stratification for post-discharge ischaemic events at a risk threshold of $4.0 \%$ led to the equivalent of 30.8 (PARIS 24.8) and 24.7 (PARIS 18.0) more true positives per 100 patients than the assumption of all patients being high risk or low risk, respectively.

\section{Predictive performance of bleeding risk scores}

Calibration of the observed probability approximated the predicted probability more closely for CREDOKyoto and PRECISE-DAPT than for PARIS (Fig. 3), which revealed some degree of underestimation and overestimation of post-discharge bleeding event rates. Discriminative capacity and reclassification are summarised in Tab. 2. PARIS-derived (c-statistic 0.55, 95\% CI 0.44-0.65) and PRECISE-DAPT-derived risk stratification (c-statistic 0.59; 95\% CI 0.48-0.69) (Fig. 4a) did not enhance risk stratification to distinguish patients with a low risk of post-discharge bleeding events from those with a high risk. CREDO-Kyoto had a moderate discriminative capacity (c-statistic 0.67, 95\% CI 0.56-0.77) to predict post-discharge bleeding events. Using PARIS as reference, the net reclassification improvement index revealed a more accurate classification with CREDO-Kyoto, but not with PRECISEDAPT (see Tab. 4 in the Electronic Supplementary Material).

Using CREDO-Kyoto-derived risk stratification, we were able to more accurately predict post-discharge bleeding events in all-comer patients with latest-generation drug-eluting stents, compared with the situation in which PARIS, PRECISE-DAPT or no model was used (Fig. 4b). Based on decision curve analysis, CREDO-Kyoto-derived risk stratification performed better than PARIS or PRECISE-DAPT. Especially when 
Table 2 Discriminative power of risk scores for post-discharge adverse events

\begin{tabular}{|c|c|c|c|}
\hline Post-discharge ischaemic risk scores & PARIS & CREDO-Kyoto & \\
\hline Hazard ratio ${ }^{a}$ & $1.15(95 \% \mathrm{Cl} 1.05$ to 1.27$)$ & $1.33(95 \% \mathrm{Cl} 1.17$ to 1.52$)$ & \\
\hline Harrell's c-statistic & $0.59(95 \% \mathrm{Cl} 0.51$ to 0.68$)$ & $0.68(95 \% \mathrm{Cl} 0.60$ to 0.75$)$ & \\
\hline Comparing c-statistic & Ref & $p=0.47^{b}$ & \\
\hline NRI (cases) & Ref & $-0.17(-0.32 \text { to }-0.19)^{c}$ & \\
\hline NRI (non-cases) & Ref & $0.28(0.25 \text { to } 0.31)^{\mathrm{c}}$ & \\
\hline Post-discharge bleeding risk scores & PARIS & CREDO-Kyoto & PRECISE-DAPT \\
\hline Hazard ratio ${ }^{\mathrm{a}}$ & $1.10(95 \% \mathrm{Cl} 0.96$ to 1.26$)$ & $1.82(95 \% \mathrm{Cl} 1.44$ to 2.31$)$ & $1.02(0.99$ to 1.05$)$ \\
\hline Harrell's c-statistic & $0.55(95 \% \mathrm{Cl} 0.44$ to 0.65$)$ & $0.67(95 \% \mathrm{Cl} 0.56$ to 0.77$)$ & 0.59 (0.48 to 0.69$)$ \\
\hline Comparing c-statistic & Ref & $p=0.075^{b}$ & $p=0.46^{b}$ \\
\hline NRI (cases) & Ref & $0.13(-0.09 \text { to } 0.34)^{c}$ & $0.06(-0.13 \text { to } 0.25)^{c}$ \\
\hline NRI (non-cases) & Ref & $0.21(0.18 \text { to } 0.24)^{c}$ & $0.11(0.08 \text { to } 0.14)^{\mathrm{c}}$ \\
\hline \multicolumn{4}{|c|}{$\begin{array}{l}\text { NRI net reclassification improvement index, } 95 \% \mathrm{Cl} 95 \% \text { confidence interval } \\
\text { aUsing risk scores (continuous variables) as a global prognostic indicator by Cox models } \\
\text { bC } \\
\text { 'Comparing c-statistic with PARIS as reference } \\
\text { 'Comparing NRI with PARIS as reference }\end{array}$} \\
\hline
\end{tabular}

a risk threshold of $\geq 2 \%$ was chosen, the net benefit of CREDO-Kyoto outperformed PARIS, PRECISE-DAPT or the assumption of all patients being high risk or low risk (see Tab. 5 in the Electronic Supplementary Material). In other words, the net benefit using CREDOKyoto at a risk threshold of $4 \%$, for example, led to the equivalent of 54.4 (PARIS 46.8 vs PRECISE-DAPT 47.5) and 8.1 more true positives per 100 patients (PARIS 0.5 vs PRECISE-DAPT 1.1) than assuming all patients as high risk or low risk, respectively.

\section{Discussion}

In this study, we assessed and directly compared the predictive performance of the PRECISE-DAPT, PARIS and CREDO-Kyoto risk scores for post-discharge events in a contemporary cohort of patients following latest-generation drug-eluting stent implantation. The main findings were: (1) CREDO-Kyoto-derived risk stratification was associated with a moderate predictive performance with respect to post-discharge events; and (2) PARIS-derived and PRECISE-DAPTderived risk stratifications had a marginal discriminative capacity to adequately define the risk of postdischarge events in unselected patients.

The discriminative capability of CREDO-Kyoto was roughly similar to that of the validation cohort (cstatistic for ischaemic events 0.68 vs 0.64 ; c-statistic for bleeding events 0.67 vs 0.66) [8]. Based on our data, CREDO-Kyoto may especially aid clinicians who need support in their clinical judgment regarding patients with a low to intermediate risk of post-discharge events. The absence of peripheral artery disease and malignancy may have introduced a slight underestimation in terms of a 1- to 2-point left-shift in the CREDO-Kyoto risk score, in approximately 10 to $15 \%$ of the patients [19]. Remarkably, this did not heavily impact the predictive capability of CREDO-Kyoto in the present analysis. The finding of a robust c-statistic while using less parameters, underscores the rele- vance of a parsimonious approach when developing a risk prediction model to optimise clinical utility.

The lower-than-expected prognostic performance of PRECISE-DAPT and PARIS for post-discharge events is largely consistent with a previous report that found a c-statistic of 0.61 for PARIS and of 0.63 for PRECISE-DAPT [20]. Noteworthy, this cohort was comprised of patients with acute coronary syndrome, whereas our cohort included roughly onehalf of patients with stable coronary artery disease and one-half of patients with acute coronary syndrome. Several issues may have contributed to the limited prognostic capability and should be borne in mind when interpreting our results. For instance, procedural parameters (i.e. chronic total occlusions, bifurcations, implantation of more than 3 stents or treatment of more than 3 lesions) were neglected in these risk scores, but are in fact related to adverse events [21, 22].

Another important limitation of currently available risk scores is that stent specific parameters are not accounted for. For example, the PRECISE-DAPT risk score was derived from a cohort in which patients were treated with $13 \%$ bare-metal stents, whereas the ReCre8 trial was comprised of a cohort that was solely treated with contemporary latest-generation drugeluting stents which may have a lower ischaemic risk profile [10].

The moderate performance of risk scores in the present study may also be explained by the composition and incidence of the incorporated risk factors [23], as related to the patient's true baseline risks. For example, none of the contemporary risk scores address the importance of lesion complexity, which also affects the risk of post-discharge events [24]. Reconciliation of such differences remains challenging and largely reflects the variety in PCI populations.

The bleeding criteria of the Bleeding Academic Research Consortium (BARC) were used to define bleeding in the present study and in the PARIS registry, as 

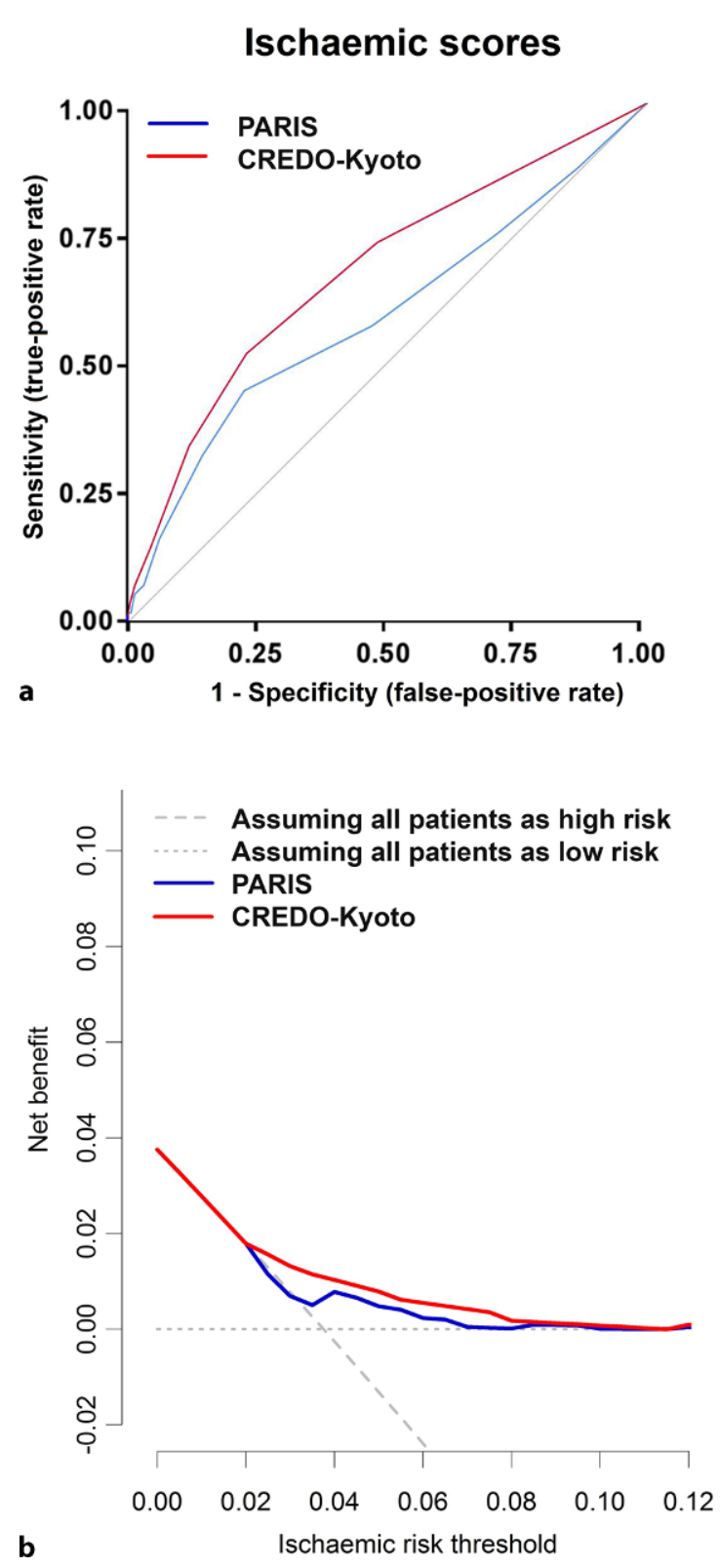

Fig. 4 Receiver operator characteristic curves (a) and decision curves (b) of contemporary risk scores. a Diagonal line indicates random classification (probability of true-positive rate being similar to probability of false-positive rate). Values above the diagonal line indicate better prediction of true positives. A larger area under the curve indicates a better discriminative

opposed to the PRECISE-DAPT study, where bleeding definitions were based on criteria for thrombolysis in myocardial infarction. This complicated the present comparison and may have underestimated the bleeding risk. The BARC criteria are, however, currently considered the standard bleeding definition [25].

Finally, rigorous testing of contemporary risk scores is essential for risk stratification, since the generalisability of risk scores remains an important drawback and is often poorly characterised. It should not be forgotten that circa 350 risk models have been investigated in cardiovascular disease over the last decades
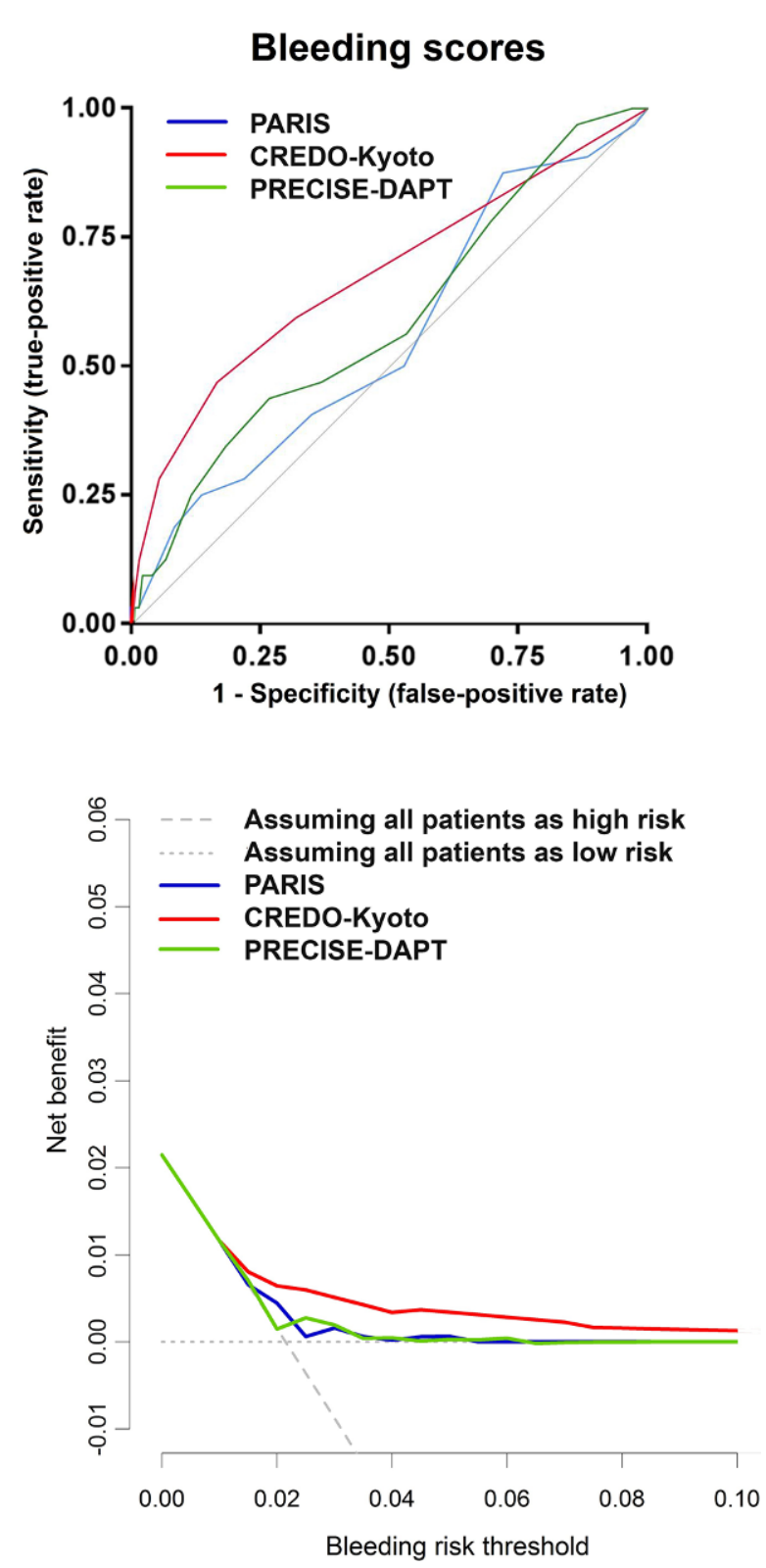

performance of the risk prediction model. b Net benefit indicates difference between true-positive and false-positive rate, corrected for threshold probability (x-axis). Higher values indicate better predictive performance of the model. Grey lines represent stratification when no model was used

[26], but that not more than a handful were resilient to independent testing [27-29]. Future studies should aim to elucidate the role of contemporary risk scores in a prospective randomised setting to evaluate their impact on clinical outcomes.

\section{Study limitations}

This study has several limitations. First, it should be noted that we addressed risk factors at the time of the index PCI. However, it seems more likely that a patient's risk is not static, but may vary over time 
[30]. Second, we obtained data over a 1-year follow-up period, whereas the risk scores were derived from data with a follow-up time of up to 3 years. This may have slightly underestimated the predictive performance of the risk scores in our cohort, although the first year after PCI is the period of greatest ischaemic and haemorrhagic risk. Third, risk stratification may have been underestimated regarding post-discharge ischaemic events and overestimated in terms of postdischarge bleeding events in troponin-negative patients because of a different regimen of DAPT. However, various DAPT strategies (e.g. 3 to 12 months) were present in the cohorts used to derive the three risk scores.

\section{Conclusion}

Based on this all-comer population treated with latest-generation drug-eluting stents, the PRECISEDAPT and PARIS risk scores were not resilient to independent testing for patients' risks of post-discharge bleeding events. CREDO-Kyoto-derived risk stratification was associated with a moderate predictive capability regarding post-discharge ischaemic or bleeding events. Future studies are warranted to improve risk assessment and to prospectively evaluate the impact on clinical outcomes when used to individualise DAPT.

Funding University Medical Centre Utrecht and participating centres.

Conflict of interest R. Rozemeijer, W.P. van Bezouwen, N.D. van Hemert, J.A. Damen, S. Koudstaal, M. Stein, G.E. Leenders, L. Timmers, A.O. Kraaijeveld, K. Roes,P. Agostoni, P.A. Doevendans, P.R. Stella and M. Voskuil declare that they have no competing interests.

Open Access This article is licensed under a Creative Commons Attribution 4.0 International License, which permits use, sharing, adaptation, distribution and reproduction in any medium or format, as long as you give appropriate credit to the original author(s) and the source, provide a link to the Creative Commons licence, and indicate if changes were made. The images or other third party material in this article are included in the article's Creative Commons licence, unless indicated otherwise in a credit line to the material. If material is not included in the article's Creative Commons licence and your intended use is not permitted by statutory regulation or exceeds the permitted use, you will need to obtain permission directly from the copyright holder. To view a copy of this licence, visit http://creativecommons.org/licenses/by/4.0/.

\section{References}

1. Yusuf S, Zhao F, Mehta SR, et al. Effects of clopidogrel in addition to aspirin in patients with acute coronary syndromes without ST-segment elevation. N Engl J Med. 2001;34:494-502.

2. WiviottSD, BraunwaldE, McCabeCH, etal. Prasugrelversus clopidogrel in patients with acute coronary syndromes. NEngl J Med. 2007;357:2001-15.
3. Wallentin L, Becker RC, Budaj A, et al. Ticagrelor versus clopidogrel in patients with acute coronary syndromes. NEngl J Med. 2009;361:1045-57.

4. Stone GW, Maehara A, Lansky AJ, et al. A prospective natural-history study of coronary atherosclerosis. N Engl J Med. 2011;364:226-35.

5. Palmerini T, Bacchi Reggiani L, Della Riva D, et al. Bleeding-related deaths in relation to the duration of dualantiplatelet therapy after coronary stenting. J Am Coll Cardiol. 2017;69:2011-22.

6. Costa F, van Klaveren D, James S, et al. Derivation and validation of the predicting bleeding complications in patients undergoing stent implantation and subsequent dual antiplatelet therapy (PRECISE-DAPT) score: a pooled analysis of individual-patient datasets from clinical trials. Lancet. 2017;389:1025-34.

7. Baber U, Mehran R, Giustino G, et al. Coronary thrombosis and major bleeding after PCI with drug-eluting stents: risk scores from PARIS. J Am Coll Cardiol. 2016;67:2224-34.

8. Natsuaki M, Morimoto T, Yamaji K, et al. Prediction of thrombotic and bleeding events after percutaneous coronary intervention: CREDO-Kyoto thrombotic and bleeding risk scores. J Am Heart Assoc. 2018; https://doi.org/10. 1161/JAHA.118.008708.

9. Rozemeijer R, Stein M, Frambach P, et al. Rationale and design of amphilimus sirolimus-eluting stents versus zotarolimus-eluting stents in all-comers requiring percutaneous coronary intervention (ReCre8): A multicenter randomized clinical trial. Catheter Cardiovasc Interv. 2018;91:410-6.

10. Rozemeijer R, Stein M, Voskuil M, et al. Randomized allcomers evaluation of a permanent polymer zotarolimuseluting stent versus a polymer-free amphilimus-eluting stent. Circulation. 2019;139:67-77.

11. Cutlip DE, Windecker S, Mehran R, et al. Clinical end points in coronary stent trials: a case for standardized definitions. Circulation. 2007;115:2344-51.

12. Mehran R, Rao SV, Bhatt DL, et al. Standardized bleeding definitions for cardiovascular clinical trials: a consensus report from the Bleeding Academic Research Consortium. Circulation. 2011;123:2736-47.

13. Collins GS, Reitsma JB, Altman DG, Moons KG. Transparent Reporting of a multivariable prediction model for Individual Prognosis or Diagnosis (TRIPOD): the TRIPOD statement. Ann Intern Med. 2015;162:55-63.

14. CockcroftDW, GaultMH. Prediction of creatinine clearance from serum creatinine. Nephron. 1976;16:31-41.

15. Astor BC, Muntner P, Levin A, Eustace JA, Coresh J. Association of kidney function with anemia: the Third National Health and Nutrition Examination Survey (1988-1994). Arch Intern Med. 2002;162:1401-8.

16. PocockSJ, Clayton TC, Altman DG. Survival plots of time-toevent outcomes in clinical trials: good practice and pitfalls. Lancet. 2002;359:1686-9.

17. DeLong ER, DeLong DM, Clarke-Pearson DL. Comparing the areas under two or more correlated receiver operating characteristic curves: a nonparametric approach. Biometrics. 1988;44:837-45.

18. Vickers AJ, Van Calster B, Steyerberg EW. Net benefit approaches to the evaluation of prediction models, molecular markers, and diagnostic tests. BMJ. 2016;352:i6.

19. Rozemeijer R, van Muiden IG, Koudstaal S, et al. One-year clinical outcomes of patients treated with polymer-free amphilimus-eluting stents or zotarolimus-eluting stents: A propensity-score adjusted analysis. Catheter Cardiovasc Interv. 2019; https://doi.org/10.1002/ccd.28041. 
20. Abu-Assi E, Raposeiras-Roubin S, Cobas-Paz R, et al. Assessing the performance of the PRECISE-DAPT and PARIS risk scores for predicting one-year out-of-hospital bleeding in acute coronary syndrome patients. EuroIntervention. 2018;13:1914-22.

21. Costa F, Van Klaveren D, Feres F, et al. Dual antiplatelet therapy duration based on ischemic and bleeding risks after coronarystenting. JAm Coll Cardiol. 2019;73:741-54.

22. Rozemeijer $R$, Wing Wong $C$, Leenders $G$, et al. Incidence, angiographic and clinical predictors, and impact of stent thrombosis: a 6-year survey of 6,545 consecutive patients. Neth Heart J. 2019; https://doi.org/10.1007/s12471-0191253-2.

23. Lloyd-Jones DM. Cardiovascular risk prediction: basic concepts, current status, and future directions. Circulation. 2010;121:1768-77.

24. Giustino G, Chieffo A, Palmerini T, et al. Efficacy and safety of dual antiplatelet therapy after complex PCI. J Am Coll Cardiol. 2016;68:1851-64.

25. Rozemeijer R, Voskuil M, Greving JP, Bots ML, Doevendans PA, Stella PR. Short versus long duration of dual antiplatelet therapy following drug-eluting stents: a metaanalysis of randomised trials. Neth Heart J. 2018;26:242-51.

26. Damen J, Hooft L, Moons KGM. Contemporary cardiovascular risk prediction. Lancet. 2018;391:1867-8.

27. Nashef SA, Roques F, Sharples LD, et al. EuroSCORE II. Eur J Cardiothorac Surg. 2012;41:734-44. discussion 44-5.

28. Gage BF, van Walraven C, Pearce L, et al. Selecting patients with atrial fibrillation for anticoagulation: stroke risk stratification in patients taking aspirin. Circulation. 2004;110:2287-92.

29. Pisters R, Lane DA, NieuwlaatR, de Vos CB, Crijns HJ, Lip GY. Anoveluser-friendly score (HAS-BLED) to assess 1-year risk of major bleeding in patients with atrial fibrillation: the Euro Heart Survey. Chest. 2010;138:1093-100.

30. Yoshida R, Ishii H, Morishima I, et al. Performance of HAS-BLED, ORBIT, PRECISE-DAPT, and PARIS risk score for predicting long-term bleeding events in patients taking an oral anticoagulant undergoing percutaneous coronary intervention. JCardiol. 2019;73:479-87. 